\title{
Superintegrable Extensions of Superintegrable Systems ${ }^{\star}$
}

\author{
Claudia M. CHANU ${ }^{\dagger}$, Luca DEGIOVANNI ${ }^{\ddagger}$ and Giovanni RASTELLI ${ }^{\S}$ \\ $\dagger$ Dipartimento di Matematica, Università di Torino, \\ Torino, via Carlo Alberto 10, Italy \\ E-mail: claudiamaria.chanu@unito.it \\ $\ddagger$ Formerly at Dipartimento di Matematica, Università di Torino, \\ Torino, via Carlo Alberto 10, Italy \\ E-mail: luca.degiovanni@gmail.com \\ $\S$ Independent researcher, cna Ortolano 7, Ronsecco, Italy \\ E-mail: giorast.giorast@alice.it
}

Received July 30, 2012, in final form September 27, 2012; Published online October 11, 2012 http://dx.doi.org/10.3842/SIGMA.2012.070

\begin{abstract}
A procedure to extend a superintegrable system into a new superintegrable one is systematically tested for the known systems on $\mathbb{E}^{2}$ and $\mathbb{S}^{2}$ and for a family of systems defined on constant curvature manifolds. The procedure results effective in many cases including Tremblay-Turbiner-Winternitz and three-particle Calogero systems.
\end{abstract}

Key words: superintegrable Hamiltonian systems; polynomial first integrals

2010 Mathematics Subject Classification: 70H06; 70H33; 53C21

\section{Introduction}

Given a natural Hamiltonian $L$ with $n$ degrees of freedom, satisfying some additional geometric conditions, it is shown in [1] how to generate a $n+1$ degrees of freedom Hamiltonian $H$, called the extension of $L$ and depending on an integer parameter $m \in \mathbb{N} \backslash\{0\}$, such that $H$ admits two new independent first integrals: $H$ itself and a polynomial in the momenta of degree $m$. This implies that, if $L$ is superintegrable with $2 n-1$ independent first integrals, then all the extended Hamiltonians $H$ also are superintegrable for any $m$ with the maximal number of $2 n+1=2(n+$ 1) - 1 first integrals, one of them of arbitrary degree $m$ whose expression is explicitly computed by means of a simple iterative process. The extension procedure, summarized in Section 2, is applied to the superintegrable systems on $\mathbb{E}^{2}$ (Section 3) and $\mathbb{S}^{2}$ (Section 5) as listed in [5]. These are all the superintegrable systems on $\mathbb{S}^{2}$ and $\mathbb{E}^{2}$ admitting two independent first integrals of degree two in the momenta other than the Hamiltonian. It is found that a great part of them admits superintegrable extensions and for some of them the extensions are explicitly determined. In Section 4 the possible natural Hamiltonians admitting an extension are determined on $\mathbb{E}^{n}$ and the superintegrable systems of Calogero and Wolfes are considered as examples in $\mathbb{E}^{3}$. In Section 6 the extension procedure is applied to a class of Hamiltonians on constant curvature manifolds to which some generalizations of the Tremblay-Turbiner-Winternitz (TTW) system belong [10]. These generalizations of the TTW systems are superintegrable for rational values of certain parameters, for which they admit polynomial first integrals of degree greater than two $[7,8]$. The cases allowing the extensions are determined and the TTW systems are among them,

${ }^{\star}$ This paper is a contribution to the Special Issue "Superintegrability, Exact Solvability, and Special Functions". The full collection is available at http://www.emis.de/journals/SIGMA/SESSF2012.html 
obtaining in this way their superintegrable extensions. Other superintegrable generalizations in higher dimensions of the TTW system are obtained in [6].

\section{Extensions of superintegrable systems}

We resume in the following statement the main results proved in [1].

Theorem 1. Let $Q$ be a n-dimensional (pseudo-)Riemannian manifold with metric tensor $\mathbf{g}$. The natural Hamiltonian $L=\frac{1}{2} g^{i j} p_{i} p_{j}+V\left(q^{i}\right)$ on $M=T^{*} Q$ with canonical coordinates $\left(p_{i}, q^{i}\right)$ admits an extension

$$
H=\frac{1}{2} p_{u}^{2}+\alpha(u) L+f(u)
$$

with a first integral $F=U^{m}(G)$ where

$$
U=p_{u}+\gamma(u) X_{L},
$$

$X_{L}$ is the Hamiltonian vector field of $L$ and $G\left(q^{i}\right)$, if and only if the following conditions hold:

i) the functions $G$ and $V$ satisfy

$$
\begin{aligned}
& \mathbf{H}(G)+m c \mathbf{g} G=\mathbf{0}, \quad m \in \mathbb{N} \backslash\{0\}, \quad c \in \mathbb{R}, \\
& \nabla V \cdot \nabla G-2 m\left(c V+L_{0}\right) G=0, \quad L_{0} \in \mathbb{R},
\end{aligned}
$$

where $\mathbf{H}(G)_{i j}=\nabla_{i} \nabla_{j} G$ is the Hessian tensor of $G$.

ii) for $c=0$ the extended Hamiltonian $H$ is

$$
H=\frac{1}{2} p_{u}^{2}+m A\left(L+V_{0}\right)+m L_{0} A^{2}\left(u+u_{0}\right)^{2},
$$

for $c \neq 0$ the extended Hamiltonian $H$ is

$$
H=\frac{1}{2} p_{u}^{2}+\frac{m\left(c L+L_{0}\right)}{S_{\kappa}^{2}\left(c u+u_{0}\right)}+W_{0},
$$

with $\kappa, u_{0}, V_{0}, W_{0}, A \in \mathbb{R}, A \neq 0$ and

$$
S_{\kappa}(x)= \begin{cases}\frac{\sin \sqrt{\kappa} x}{\sqrt{\kappa}}, & \kappa>0, \\ x, & \kappa=0, \\ \frac{\sinh \sqrt{|\kappa|} x}{\sqrt{|\kappa|}}, & \kappa<0 .\end{cases}
$$

Dynamically, extended Hamiltonians (1) can be written as

$$
\frac{1}{2} p_{u}^{2}-\frac{m}{S_{\kappa}^{2}\left(c u+u_{0}\right)} \eta-h=0, \quad c L+L_{0}+\eta=0, \quad \text { if } \quad c \neq 0,
$$

and

$$
\frac{1}{2} p_{u}^{2}+m L_{0} A^{2} u^{2}-\eta-h=0, \quad m A\left(L+V_{0}\right)+\eta=0, \quad \text { if } \quad c=0
$$


with $H=h$, and where constant $\eta$ can be understood either as a separation constant, between the elements of $H$ depending on $\left(u, p_{u}\right)$ and those depending on $\left(q^{i}, p_{i}\right)$, or as a coupling constant merging together the Hamiltonian $L$ on $T^{*} Q$ and the Hamiltonian

$$
\frac{1}{2} p_{u}^{2}-\frac{m}{S_{\kappa}^{2}\left(c u+u_{0}\right)}, \quad c \neq 0, \quad \text { or } \quad \frac{1}{2} p_{u}^{2}+m L_{0} A^{2} u^{2}, \quad c=0,
$$

depending on $\left(u, p_{u}\right)$, to build the extended Hamiltonian $H$. Several examples are given in [1].

Under the hypotheses of Theorem 1 , recalling that $C_{\kappa}(x)=\frac{d}{d x} S_{\kappa}(x)$, the polynomials in the momenta

$$
U^{m} G= \begin{cases}\left(p_{u}+\frac{C_{\kappa}\left(c u+u_{0}\right)}{S_{\kappa}\left(c u+u_{0}\right)} X_{L}\right)^{m} G, & c \neq 0, \\ \left(p_{u}-A\left(u+u_{0}\right) X_{L}\right)^{m} G, & c=0,\end{cases}
$$

are first integrals of $H$ of degree $m$. For example,

$$
U G=G p_{u}+\gamma(u) X_{L}(G)=G p_{u}+\gamma(u)\{L, G\}
$$

where $\{$,$\} are the Poisson brackets. Another way to calculate U^{m} G$ is to apply the formula [2]

$$
U^{m} G=P_{m} G+D_{m} X_{L} G
$$

with

$$
\begin{aligned}
P_{m} & =\sum_{k=0}^{[m / 2]}\left(\begin{array}{c}
m \\
2 k
\end{array}\right) \gamma^{2 k} p_{u}^{m-2 k}\left(-2 m\left(c L+L_{0}\right)\right)^{k}, \\
D_{m} & =\sum_{k=0}^{[m / 2]-1}\left(\begin{array}{c}
m \\
2 k+1
\end{array}\right) \gamma^{2 k+1} p_{u}^{m-2 k-1}\left(-2 m\left(c L+L_{0}\right)\right)^{k}, \quad m>1,
\end{aligned}
$$

where $[\cdot]$ denotes the integer part, $D_{1}=\gamma$ and

$$
\gamma(u)= \begin{cases}\frac{C_{\kappa}\left(c u+u_{0}\right)}{S_{\kappa}\left(c u+u_{0}\right)}, & c \neq 0, \\ -A\left(u+u_{0}\right), & c=0 .\end{cases}
$$

Remark 1. For $c=0$, if $L_{0}=0$ then $p_{u}$ is a first integral of the extended Hamiltonian and the extended potential is merely $m A V$, with $m$ and $A$ constants. Therefore the extension is trivial. Otherwise, a harmonic oscillator term in the variable $u$, attractive or repulsive, is added to the potential $m A V$. In the case $c \neq 0$, we remark that, in the extended Hamiltonian (5), the potential $V$ is multiplied by a non-constant factor depending on $u$.

Remark 2. The expression (6) of $\gamma(u)$ is determined in [1] as the general solution of the differential equation

$$
\gamma^{\prime}+c\left(\gamma^{2}+\kappa\right)=0
$$

for real values of $\gamma, u, c$ and $\kappa$. However, the general solution of equation (7) in the complex case can be considered too. Its expression is the same as (6) if we extend functions $S_{\kappa}$ and $C_{\kappa}$ to complex values of $x$ and $\kappa$ by using the standard exponential expressions of trigonometric functions. After this generalization, the extension procedure characterized by Theorem 1 can be applied also to the complex case. 
In [1] it is proved that, if $n>1$, then (2) admits a complete solution $G$ depending on a maximal number of parameters $\left(a_{i}\right)$, with $i=0, \ldots, n$, iff the sectional curvature of $Q$ is constant and equal to $m c$. Once such a solution $G$ is known, an extension of $L$ is possible iff the compatibility condition (3) on $V$ is satisfied. A sharper result on constant curvature manifolds is the following

Proposition 1. On a manifold with constant curvature $K$ the only eigenvalues $m c$ for the Hessian equation (2) are either zero or the curvature $K$. Moreover, if $K \neq 0$ and $m c=0$ then the only solutions of (2) are $G=$ const.

Proof. The equation (2), written in components, becomes

$$
\nabla_{i} \nabla_{j} G+m c g_{i j} G=\partial_{i j} G-\Gamma_{i j}^{k} \partial_{k} G+m c g_{i j} G=0 .
$$

and the integrability conditions that each solution $G\left(q^{i}\right)$ must satisfy are given by (see [1] for details)

$$
R_{h i j}^{k} z_{k}=m c\left(g_{j h} z_{i}-g_{i h} z_{j}\right), \quad \forall h, \quad \forall i \neq j,
$$

where $z_{k}=\partial_{k} G / G$ and $R_{h i j}^{k}=\partial_{i} \Gamma_{j l}^{k}-\partial_{j} \Gamma_{i l}^{k}+\Gamma_{j l}^{h} \Gamma_{i h}^{k}-\Gamma_{i l}^{h} \Gamma_{j h}^{k}$ is the Riemann tensor of the metric. For a constant curvature manifold we have $R_{h l i j}=K\left(g_{j l} g_{h i}-g_{i l} g_{h j}\right)$ and the above conditions become

$$
(K-m c)\left(g_{j h} z_{i}-g_{i h} z_{j}\right)=0, \quad \forall h, \quad \forall i \neq j .
$$

By choosing orthogonal coordinates, we see that, since $i \neq j$, the equations are identically satisfied for $h \neq i, j$. Otherwise, they reduce to $(K-m c) g_{j j} z_{i}=0$. Hence, for $m c \neq K$, the only possibility is $z_{i}=0$ for all $i$ (that is, $G$ is a constant). For $m c \neq K$ and $m c \neq 0$, by (2) we get $G=0$, thus $m c$ is not an eigenvalue.

In [1] it is shown that the first integral $U^{m}(G)$ is functionally independent from $H, L$ and all its possible first integrals $L_{i}$ in $T^{*} Q$. It is straightforward to see that $L$ and $L_{i}$ are first integrals of $H$, therefore, if $L$ is a superintegrable Hamiltonian with $2 n-1$ first integrals, inclu$\operatorname{ding} L$, then $H$ is superintegrable too with $2 n-1+2=2(n+1)-1$ first integrals including $H$ itself. It follows that the extension procedure applied to a superintegrable Hamiltonian $L$, under the hypothesis of Theorem 1, always produces a new superintegrable Hamiltonian $H$. Given a superintegrable system of Hamiltonian $L=\frac{1}{2} g^{i j} p_{i} p_{j}+V\left(q^{i}\right)$ with a configuration manifold $Q$ of constant curvature $K$, its extension to another superintegrable system of Hamiltonian $H$, when possible, can be obtained by applying the following algorithm (see also [1]):

1. Solve equation (2) on the manifold $Q$, with $c=K / m$, to compute the general form of the function $G\left(q^{i}, a_{0}, \ldots, a_{n}\right)$.

2. Solve equation (3) with the given $V$ for some of the parameters $\left(a_{i}\right)$ in $G$. This is a crucial step, because if no solution is found, except for the trivial one $G=0$, then the extension is not possible.

3. Determine the extension via Theorem 1 .

4. Compute $U^{m}(G)$ to obtain the additional first integral.

In the following sections we analyze several examples of extensions of superintegrable systems. 


\section{Superintegrable extensions of $\mathbb{E}^{2}$ systems}

Since the curvature of $\mathbb{E}^{2}$ is zero, equation (2) admits, by Proposition 1 , solutions $G \neq 0$ only for $m c=0$. Thus, we are in the case $c=0$ of Theorem 1 and the extended Hamiltonian $H$ is in the form (4). In [1] the complete solution $G$ of (2) is computed, in standard Cartesian coordinates of $\mathbb{E}^{2}$, as

$$
G=a_{0}+a_{1} x+a_{2} y
$$

Equation (3) becomes here

$$
\partial_{x} V \partial_{x} G+\partial_{y} V \partial_{y} G=2 m L_{0} G
$$

whose general solution is

$$
V=m L_{0}\left[\left(x+x^{0}\right)^{2}+\left(y+y^{0}\right)^{2}\right]+F\left(a_{2} x-a_{1} y\right),
$$

with the constraint $a_{0}=a_{1} x^{0}+a_{2} y^{0}$, where $x^{0}, y^{0} \in \mathbb{R}$ or $\mathbb{C}$ and $F$ is any regular function of the argument. The extension, after the non restrictive assumptions $V_{0}=u_{0}=0$ and $A=m^{-1}$, becomes

$$
H=\frac{1}{2} p_{u}^{2}+L+\frac{L_{0}}{m} u^{2}=\frac{1}{2}\left(p_{u}^{2}+p_{x}^{2}+p_{y}^{2}\right)+V+\frac{L_{0}}{m} u^{2} .
$$

In [5] the list of all superintegrable potentials in $\mathbb{E}^{2}$ with three independent quadratic in the momenta first integrals is given, up to isometries and reflections. In that article $\mathbb{E}^{2}$ is assumed to be a two-dimensional complex manifold. According to Remark 2 we apply in this case the same procedure developed for the real case and allow all functions, variables and parameters (except for $m \in \mathbb{N}$ ) to take indifferently real or complex values, in this one and all the following sections. By setting $z=x+i y, \bar{z}=x-i y$ (remark that, despite the notation, if $x$ and $y$ are complex coordinates, then $z$ and $\bar{z}$ are not complex conjugate one of the other), the list is

$$
\begin{array}{ll}
E 1 \quad V=\frac{\alpha_{1}}{x^{2}}+\frac{\alpha_{2}}{y^{2}}+\alpha_{3}\left(x^{2}+y^{2}\right), \\
E 2 \quad V=\alpha_{1} x+\frac{\alpha_{2}}{y^{2}}+\alpha_{3}\left(4 x^{2}+y^{2}\right), \\
E 3 \quad V=\alpha_{3}\left(x^{2}+y^{2}\right), \\
E 4 \quad V=\alpha_{1}(x+i y), \\
E 5 \quad V=\alpha_{1} x \\
E 6 \quad V=\frac{\alpha_{1}}{x^{2}} \\
E 7 \quad V=\frac{\alpha_{1} \bar{z}}{\sqrt{\bar{z}^{2}-k^{2}}}+\frac{\alpha_{2} z}{\sqrt{\bar{z}^{2}-k^{2}}\left(\bar{z}+\sqrt{\bar{z}^{2}-k^{2}}\right)^{2}}+\alpha_{3} z \bar{z} \\
E 8 & V=\frac{\alpha_{1} z}{\bar{z}^{3}}+\frac{\alpha_{2}}{\bar{z}^{2}}+\alpha_{3} z \bar{z}, \\
E 9 & V=\frac{\alpha_{1}}{\sqrt{\bar{z}}+\alpha_{2} x+\alpha_{3} \frac{x+\bar{z}}{\sqrt{\bar{z}}},} \\
E 10 & V=\alpha_{1} \bar{z}+\alpha_{2}\left(z-\frac{3}{2} \bar{z}^{2}\right)+\alpha_{3}\left(z \bar{z}-\frac{1}{2} \bar{z}^{3}\right) \\
E 11 & V=\alpha_{1} z+\frac{\alpha_{2} z}{\sqrt{\bar{z}}}+\frac{\alpha_{3}}{\sqrt{\bar{z}}}, \\
E 12 & V=\frac{\alpha_{1} \bar{z}}{\sqrt{\bar{z}^{2}+k^{2}}},
\end{array}
$$




$$
\begin{aligned}
& E 13 \quad V=\frac{\alpha_{1}}{\sqrt{\bar{z}}} \\
& E 14 \quad V=\frac{\alpha_{1}}{\bar{z}^{2}} \\
& E 15 V=h(\bar{z}), \quad \text { for any function } h, \\
& E 16 \quad V=\frac{1}{\sqrt{x^{2}+y^{2}}}\left(\alpha_{1}+\frac{\alpha_{2}}{x+\sqrt{x^{2}+y^{2}}}+\frac{\alpha_{3}}{x-\sqrt{x^{2}+y^{2}}}\right) \text {, } \\
& E 17 \quad V=\frac{\alpha_{1}}{\sqrt{z \bar{z}}}+\frac{\alpha_{2}}{z^{2}}+\frac{\alpha_{3}}{z \sqrt{z \bar{z}}}, \\
& E 18 \quad V=\frac{\alpha_{1}}{\sqrt{x^{2}+y^{2}}}, \\
& E 19 \quad V=\frac{\alpha_{1} \bar{z}}{\sqrt{\bar{z}^{2}-4}}+\frac{\alpha_{2}}{\sqrt{z(\bar{z}+2)}}+\frac{\alpha_{3}}{\sqrt{z(\bar{z}-2)}}, \\
& E 20 \quad V=\frac{1}{\sqrt{x^{2}+y^{2}}}\left(\alpha_{1}+\alpha_{2} \sqrt{x+\sqrt{x^{2}+y^{2}}}+\alpha_{3} \sqrt{x-\sqrt{x^{2}+y^{2}}}\right) \text {, }
\end{aligned}
$$

with $\left(\alpha_{i}\right), k \in \mathbb{C}$. The equation of compatibility (3) is equivalent to a linear homogeneous expression in $\left(a_{i}\right)$ with coefficients linear but not homogeneous in $\alpha_{i}$. This expression vanishes only for some suitable choices of the parameters $a_{i}$ and $\alpha_{i}$, depending on the extension parameters $m, L_{0}$. The solution (9) of (3) shows that a non null function $G$ of the form (8) satisfying (3) exists only for the following potentials

\begin{tabular}{|c|l|c|c|}
\hline & \multicolumn{1}{|c|}{$V$} & $G$ & particular cases of \\
\hline$i$ & $m L_{0}\left(x^{2}+y^{2}\right)$ & $a_{1} x+a_{2} y$ & $E 1, E 3, E 7, E 8$ \\
$i i$ & $\frac{\alpha_{1}}{x^{2}}+m L_{0}\left(x^{2}+y^{2}\right)$ & $a_{2} y$ & $E 1$ \\
$i i i$ & $\frac{\alpha_{2}}{y^{2}}+m L_{0}\left(x^{2}+y^{2}\right)$ & $a_{1} x$ & $E 1$ \\
$i v$ & $\alpha_{1} x+m L_{0}\left(4 x^{2}+y^{2}\right)$ & $a_{2} y$ & $E 2$ \\
$v$ & $\alpha_{1} x+\frac{\alpha_{2}}{y^{2}}+\frac{m L_{0}}{4}\left(4 x^{2}+y^{2}\right)$ & $a_{1}\left(\frac{\alpha_{1}}{2 m L_{0}}+x\right)$ & $E 2$ \\
$v i$ & $\frac{\alpha_{1} \bar{z}}{\sqrt{\bar{z}^{2}-k^{2}}+m L_{0}\left(x^{2}+y^{2}\right)}$ & $a_{1} \bar{z}$ & $E 7$ \\
vii & $\frac{\alpha_{2}}{\bar{z}^{2}}+m L_{0}\left(x^{2}+y^{2}\right)$ & $a_{1} \bar{z}$ & $E 8$ \\
viii & $\alpha_{1} \bar{z}+\alpha_{2}\left(z-\frac{3}{2} \bar{z}^{2}\right)+m L_{0}\left(z \bar{z}-\frac{\bar{z}^{3}}{2}\right)$ & $a_{1}\left(\frac{\alpha_{2}}{m L_{0}}+\bar{z}\right)$ & $E 10$ \\
\hline
\end{tabular}

The potentials admitting $G\left(a_{i}\right)$ depending on several $a_{i}$ allow the existence of different first integrals of the form $U^{m} G\left(a_{i}\right)$. However, since the system is already superintegrable by including a single $U^{m} G\left(a_{i}\right)$, all the other first integrals obtained in this way functionally depend on the known ones.

Because $L_{0} \neq 0$, a necessary condition for the extensibility is the presence of a harmonic term in the potential $V$.

\section{Extensions of the harmonic oscillators}

As an example, we analyze into details the extensions of the isotropic harmonic oscillator (a particular case of $E 1, E 3, E 7, E 8$ )

$$
V_{i}=\alpha_{3}\left(x^{2}+y^{2}\right)
$$


and of the anisotropic one (a particular case of $E 2$ )

$$
V_{a}=\alpha_{3}\left(4 x^{2}+y^{2}\right) .
$$

In both cases we assume $\alpha_{3} \neq 0$ to avoid a trivial potential.

The only possible extension for $V_{i}$ is

$$
\begin{aligned}
H & =\frac{1}{2}\left(p_{u}^{2}+p_{x}^{2}+p_{y}^{2}\right)+\alpha_{3}\left(x^{2}+y^{2}\right)+\frac{L_{0}}{m} u^{2} \\
& =\frac{1}{2}\left(p_{u}^{2}+p_{x}^{2}+p_{y}^{2}\right)+\alpha_{3}\left(x^{2}+y^{2}+\frac{u^{2}}{m^{2}}\right),
\end{aligned}
$$

with the constraint $\alpha_{3}-m L_{0}=0$ due to (3) that sets the potential in the tabulated form. The Hamiltonian (11) represents an anisotropic oscillator in $\mathbb{E}^{3}$ and shows explicitly its superintegrability being $m$ an integer. Recalling that $U=p_{u}-m^{-1} u X_{L}$, and by setting $X_{L} G=$ $a_{1} p_{x}+a_{2} p_{y}=P$, we obtain as an example the expression of $U^{4} G$

$$
U^{4} G=G p_{u}^{4}-u p_{u}^{3} P-\frac{3}{4} a_{3} G u^{2} p_{u}^{2}+\frac{a_{3}}{8} u^{3} p_{u} P+\frac{a_{3}^{2}}{64} G u^{4} .
$$

The anisotropic oscillator (10) admits, instead, two extensions, corresponding to the two different functions $G$ and two different relations between $\alpha_{3}$ and $L_{0}$ (items $(i v)$ and $(v)$ of the table with $\alpha_{1}=\alpha_{2}=0$ ). In order to analyze this case in full generality, an iterated procedure of extension can be applied. By starting from the one-dimensional oscillator

$$
H_{1}=\frac{1}{2} p_{1}^{2}+\omega x_{1}^{2},
$$

we build a first extension

$$
H_{2}=\frac{1}{2} p_{2}^{2}+H_{1}+\frac{\omega}{m_{1}^{2}} x_{2}^{2}=\frac{1}{2}\left(p_{1}^{2}+p_{2}^{2}\right)+\omega x_{1}^{2}+\frac{\omega}{m_{1}^{2}} x_{2}^{2}
$$

where we use $x_{2}=u$ and, because of (3), we have $G_{1}=a_{1} x_{1}$ and $L_{0}=\omega / m_{1}$. The resulting potential is an anisotropic oscillator and our procedure produces the third first integral of degree $m_{1}$ in the momenta $U^{m_{1}} G_{1}=\left(p_{2}-m_{1}^{-1} X_{H_{1}}\right)^{m_{1}} G_{1}$. The potential of $H_{2}$ coincides with $V_{a}$ for $m_{1}=2$ and $\omega=4 \alpha_{3}$ (this is the unique case with a third quadratic first integral).

A further step is the research of an extension of $H_{2}$

$$
H_{3}=\frac{1}{2}\left(p_{1}^{2}+p_{2}^{2}+p_{3}^{2}\right)+\omega x_{1}^{2}+\frac{\omega}{m_{1}^{2}} x_{2}^{2}+\frac{L_{0}}{m_{2}} x_{3}^{2},
$$

where the value of $L_{0}$ has to be determined. In this case, since the general $G_{2}$ is (8), condition (3) becomes

$$
a_{1} \omega x_{1}+a_{2} \frac{\omega}{m_{1}^{2}} x_{2}=m_{2} L_{0}\left(a_{0}+a_{1} x_{1}+a_{2} x_{2}\right) .
$$

Hence, we get $a_{0}=0$, and

$$
a_{1}\left(\omega-m_{2} L_{0}\right)=0, \quad a_{2}\left(\frac{\omega}{m_{1}^{2}}-m_{2} L_{0}\right)=0 .
$$

If $a_{1}=0$, we need $a_{2} \neq 0$ and

$$
L_{0}=\frac{\omega}{m_{1}^{2} m_{2}}, \quad G_{2}=a_{2} x_{2} .
$$


If $a_{1} \neq 0$ and $a_{2}=0$, then we have

$$
L_{0}=\frac{\omega}{m_{2}}, \quad G_{2}=a_{1} x_{1} .
$$

Finally, if both $a_{1} \neq 0$ and $a_{2} \neq 0$ the two conditions are satisfied iff $m_{1}^{2}=1$ (i.e., $H_{2}$ is the Hamiltonian of an isotropic oscillator) and

$$
L_{0}=\frac{\omega}{m_{2}}, \quad G_{2}=a_{1} x_{1}+a_{2} x_{2} .
$$

In the first two cases, $H_{3}$ represents an anisotropic harmonic oscillator, which is superintegrable because $m_{1}, m_{2}$ are integers, but they have different functions $G_{2}$ and consequently different first integrals $U^{m_{2}} G_{2}=\left(p_{3}-m_{2}^{-1} X_{H_{2}}\right)^{m_{2}} G_{2}$. If we set $m_{1}=2, m_{2}=m$ and $\omega=4 \alpha_{3}$ in order to restrict ourselves to the potential (10), we obtain two possible extensions: if $a_{1}=0$ we have the relation $\alpha_{3}-m L_{0}=0$ that gives the first form in the table. If, otherwise, $a_{2}=0$ we have the relation $4 \alpha_{3}-m L_{0}=0$ that gives the second one.

The extension procedure can be iterated indefinitely obtaining at the $n$-th step an $n$-dimensional anisotropic oscillator with a complete set of first integrals $\left(U^{m_{1}} G_{1}, U^{m_{2}} G_{2}, \ldots, U^{m_{n-1}} G_{n}\right)$ of degree $\left(m_{1}, m_{2}, \ldots, m_{n-1}\right)$, that, together with the $n$ Hamiltonians $\left(H_{1}, H_{2}, \ldots, H_{n}\right)$ make the system superintegrable. We remark that the systems obtained in this way are characterized by the fact that the frequencies are all integer multiples of one of them. See $[4,9]$ for additional details on superintegrability of anisotropic oscillators.

\section{Superintegrable extensions of $\mathbb{E}^{n}$}

It is straightforward to generalize to $\mathbb{E}^{n}$ the procedure previously applied to $\mathbb{E}^{2}$. Let us consider in $\mathbb{E}^{n}$ with Cartesian coordinates the Hamiltonian

$$
L=\frac{1}{2}\left(p_{1}^{2}+p_{2}^{2}+\cdots+p_{n}^{2}\right)+V\left(x_{1}, x_{2}, \ldots, x_{n}\right) .
$$

The general solution of (2) and (3) are

$$
\begin{aligned}
& G=a_{0}+a_{1} x_{1}+a_{2} x_{2}+\cdots+a_{n} x_{n} \\
& V=m L_{0}\left[\left(x_{1}+x_{1}^{0}\right)^{2}+\cdots+\left(x_{n}+x_{n}^{0}\right)^{2}\right]+F\left(a_{1} x_{2}-a_{2} x_{1}, \ldots, a_{1} x_{n}-a_{n} x_{1}\right),
\end{aligned}
$$

with the constraint $a_{0}=\sum_{i=1}^{n} a_{i} x_{i}^{0}$, where $x_{i}^{0} \in \mathbb{R}$ or $\mathbb{C}, F$ is any regular function of the arguments and $L_{0} \neq 0$. The corresponding extension is

$$
H=\frac{1}{2} p_{u}^{2}+m A\left(L+V_{0}\right)+m L_{0} A^{2}\left(u+u_{0}\right)^{2} .
$$

We remark that, as well as in dimension 2, the presence of a harmonic term in $V$ is a necessary condition for the extensibility.

\section{Extensions of the three-body Calogero and Wolfes systems}

We consider the particular case of $n=3$. If $a_{2}=a_{3}=a_{1}$ and $F\left(X_{1}, X_{2}\right)$ in (12) is

$$
F=k\left(X_{1}^{-2}+X_{2}^{-2}+\left(X_{1}-X_{2}\right)^{-2}\right),
$$

with $k \in \mathbb{R}$, then

$$
F=k\left(\frac{1}{(x-y)^{2}}+\frac{1}{(x-z)^{2}}+\frac{1}{(y-z)^{2}}\right)
$$


coincides with the celebrated Calogero potential, which is a well known superintegrable system (see for example [3] and references therein). If, with the same choice for the $a_{i}$,

$$
F=k\left(\left(X_{1}+X_{2}\right)^{-2}+\left(2 X_{1}-X_{2}\right)^{-2}+\left(2 X_{2}-X_{1}\right)^{-2}\right),
$$

then $F$ coincides with the Wolfes potential, a three-body superintegrable interaction whose dynamic equivalence with the Calogero potential is discussed in [3]. If $L_{0} \neq 0$, then the first integrals of the extended Hamiltonian for $m=2,3$ and $F$ in the Calogero form are respectively

$$
\begin{aligned}
& U^{2} G=G p_{u}^{2}-2 A u p_{u} P-4 A^{2} L_{0} G u^{2}, \\
& U^{3} G=G p_{u}^{3}-3 A u p_{u}^{2} P-18 A^{2} L_{0} G u^{2} p_{u}+6 A^{3} L_{0} u^{3} P,
\end{aligned}
$$

where $G=\left(x_{1}^{0}+x_{2}^{0}+x_{3}^{0}+x_{1}+x_{2}+x_{3}\right)$ and $P=X_{L}(G)=p_{1}+p_{2}+p_{3}$ is the conserved linear momentum.

\section{$5 \quad$ Superintegrable extensions of $\mathbb{S}^{2}$}

In [1] the complete solution $G$ of (2) for $m c=1$ (the constant curvature of a sphere of radius 1 ) is computed in standard spherical coordinates as

$$
G=a_{0} \cos \theta+\left(a_{1} \sin \phi+a_{2} \cos \phi\right) \sin \theta .
$$

Equation (3) becomes

$$
\partial_{\theta} V \partial_{\theta} G+\frac{1}{\sin ^{2} \theta} \partial_{\phi} V \partial_{\phi} G=2 V G .
$$

Therefore, $c=\frac{1}{m} \neq 0$ and, by Theorem 1 , the extension of $L$ is

$$
\frac{1}{2} p_{u}^{2}+\frac{1}{S_{\kappa}^{2}\left(\frac{1}{m} u\right)}\left(\frac{1}{2}\left(p_{\theta}^{2}+\frac{1}{\sin ^{2} \theta} p_{\phi}^{2}\right)+V\right),
$$

where we assume without restrictions the constants $u_{0}, L_{0}$ and $W_{0}$ all zero, and where $\kappa \in \mathbb{R}$. Since $|\kappa|$ can be multiplied by a positive constant simply by rescaling $u$, we can assume, if $\kappa \neq 0$, $|\kappa|=m^{2}$ so that the extensions become

$$
\begin{aligned}
H_{m}^{+}=\frac{1}{2} p_{u}^{2}+\frac{m^{2}}{\sin ^{2} u}\left(\frac{1}{2}\left(p_{\theta}^{2}+\frac{1}{\sin ^{2} \theta} p_{\phi}^{2}\right)+V\right), & & \kappa>0, \\
H_{m}^{0}=\frac{1}{2} p_{u}^{2}+\frac{m^{2}}{u^{2}}\left(\frac{1}{2}\left(p_{\theta}^{2}+\frac{1}{\sin ^{2} \theta} p_{\phi}^{2}\right)+V\right), & & \kappa=0, \\
H_{m}^{-}=\frac{1}{2} p_{u}^{2}+\frac{m^{2}}{\sinh ^{2} u}\left(\frac{1}{2}\left(p_{\theta}^{2}+\frac{1}{\sin ^{2} \theta} p_{\phi}^{2}\right)+V\right), & & \kappa<0 .
\end{aligned}
$$

For $\kappa \in \mathbb{C}$, the explicit form of the extension follows from Remark 2 .

We remark that the numerical factor $m^{2}$ can be absorbed into $L$ by a rescaling of the coordinates $(\theta, \phi)$. Whenever $V$ depends on $(\theta, \phi)$ only through trigonometric functions, the rescaling enlights the existence of discrete (polyhedral) symmetries of $L$ on $\mathbb{S}^{2}$ of order depending on $m$.

The superintegrable potentials on $\mathbb{S}^{2}$ with first integrals all quadratic in the momenta are determined in [5], where the sphere is intended, as $\mathbb{E}^{2}$ previously, as a complex manifold. The nine different superintegrable potentials, up to symmetries in $O(3, \mathbb{C})$ including reflections, are, in Cartesian three-dimensional coordinates $(x, y, z)$ with $x=\sin \theta \cos \phi, y=\sin \theta \sin \phi$, $z=\cos \theta[5]$

$$
S 1 \quad V=\frac{\alpha_{1}}{\bar{w}^{2}}+\frac{\alpha_{2} z}{\bar{w}^{3}}+\frac{\alpha_{3}\left(1-4 z^{2}\right)}{\bar{w}^{4}},
$$




$$
\begin{array}{ll}
S 2 \quad V & =\frac{\alpha_{1}}{z^{2}}+\frac{\alpha_{2}}{\bar{w}^{2}}+\frac{\alpha_{3} w}{\bar{w}^{3}}, \\
S 3 \quad V & =\frac{\alpha_{1}}{z^{2}}, \\
S 4 \quad V & =\frac{\alpha_{1}}{\bar{w}^{2}}+\frac{\alpha_{2} z}{\sqrt{x^{2}+y^{2}}}+\frac{\alpha_{3}}{\bar{w} \sqrt{x^{2}+y^{2}}}, \\
S 5 \quad V & =\frac{\alpha_{1}}{\bar{w}^{2}}, \\
S 6 \quad V & =\frac{\alpha_{1} z}{\sqrt{x^{2}+y^{2}}}, \\
S 7 & V=\frac{\alpha_{1} x}{\sqrt{y^{2}+z^{2}}}+\frac{\alpha_{2} y}{z^{2} \sqrt{y^{2}+z^{2}}}+\frac{\alpha_{3}}{z^{2}}, \\
S 8 & V=\frac{\alpha_{1} x}{\sqrt{y^{2}+z^{2}}}+\frac{\alpha_{2}(w-z)}{\sqrt{w(z-i y)}}+\frac{\alpha_{3}(w+z)}{\sqrt{w(z+i y)}}, \\
S 9 & V=\frac{\alpha_{1}}{x^{2}}+\frac{\alpha_{2}}{y^{2}}+\frac{\alpha_{3}}{z^{2}},
\end{array}
$$

where $w=x+i y, \bar{w}=x-i y, \alpha_{1}, \alpha_{2}, \alpha_{3} \in \mathbb{C}$. We remark that in $(x, y, z)$ we have

$$
G=a_{2} x+a_{1} y+a_{0} z
$$

with $a_{0}, a_{1}, a_{2} \in \mathbb{C}$.

By putting the previous expressions for $V$ and $G$ given by (13) into (3), we get a linear homogeneous function in $\left(a_{i}\right)$ whose components are linear homogeneous in $\left(\alpha_{1}, \alpha_{2}, \alpha_{3}\right)$. The non-trivial solutions (with $V$ and $G$ both not constant) of the equation are

\begin{tabular}{|c|c|c|c|}
\hline & $V$ & $G$ & particular cases of \\
\hline$i$ & $\frac{\alpha_{2}}{\bar{w}^{2}}+\frac{\alpha_{3} w}{\bar{w}^{3}}$ & $a_{0} z$ & $S 2$ \\
$i i$ & $\frac{\alpha_{1}}{\bar{w}^{2}}+\frac{\alpha_{2}}{\bar{w} \sqrt{x^{2}+y^{2}}}$ & $a_{0} z$ & $S 4$ \\
$i i i$ & $\frac{\alpha_{1}}{\bar{w}^{2}}$ & $a_{0} z$ & $S 1, S 2, S 4, S 5$ \\
$i v$ & $\frac{\alpha_{1}}{z^{2}}+\frac{\alpha_{2} y}{z^{2} \sqrt{y^{2}+z^{2}}}$ & $a_{2} x$ & $S 7$ \\
$v$ & $\frac{\alpha_{1}}{z^{2}}$ & $a_{2} x+a_{1} y$ & $S 2, S 3, S 7, S 9$ \\
$v i$ & $\frac{\alpha_{1}}{x^{2}}+\frac{\alpha_{2}}{y^{2}}$ & $a_{0} z$ & $S 9$ \\
\hline
\end{tabular}

The only cases without superintegrable extensions for any combination of parameters are $S 6$ and $S 8$, that are strictly related. The extensible cases of $S 9$ are all equivalent to $(v)$ or $(v i)$ up to permutation of the coordinates. Case $(v)$ can be considered a subcase of $(i v)$, but it is listed apart because the corresponding expression of $G$ is different.

\section{Extensions of $S 9$}

As an example of the extension procedure, we develop the computations of extensions and first integrals for the case $S 9$. For the subcase $(v)$ we have $V=\frac{\alpha_{3}}{\cos ^{2} \theta}$, with $G=\left(a_{1} \sin \phi+\right.$ $\left.a_{2} \cos \phi\right) \sin \theta$. For $m=3$ we have for the Hamiltonian $H_{3}^{+}$the first integral

$$
U^{3} G=B \sin \theta p_{u}^{3}-27 B \operatorname{ctan}^{3} u \cos \theta p_{\theta}^{3}-27 C \frac{\operatorname{ctan}^{3} u}{\sin ^{3} \theta} p_{\phi}^{3}+B \cos \theta \operatorname{ctan} u p_{u}^{2} p_{\theta}
$$




$$
\begin{aligned}
& +9 C \frac{\operatorname{ctan} u}{\sin \theta} p_{u}^{2} p_{\phi}-27 B \operatorname{ctan}^{2} u \sin \theta p_{u} p_{\theta}^{2}-27 B \frac{\operatorname{ctan}^{2} u}{\sin \theta} p_{u} p_{\phi}^{2}-27 C \frac{\operatorname{ctan}^{3} u}{\sin \theta} p_{\theta}^{2} p_{\phi} \\
& -27 B \frac{\operatorname{ctan}^{3} u \cos \theta}{\sin ^{2} \theta} p_{\phi}^{2} p_{\theta}-54 \alpha_{3}\left(B \frac{\operatorname{ctan}^{2} u \sin \theta}{\cos \theta} p_{u}+B \frac{\operatorname{ctan}^{3} u}{\cos \theta} p_{\theta}+C \frac{\operatorname{ctan}^{3} u}{\sin \theta \cos ^{2} \theta} p_{\phi}\right),
\end{aligned}
$$

where $B=a_{1} \sin \phi+a_{2} \cos \phi, C=a_{1} \cos \phi-a_{2} \sin \phi=\frac{d B}{d \phi}$.

The second of the subcases of $S 9$ admitting a superintegrable extension is $(v i)$

$$
V=\frac{1}{\sin ^{2} \theta}\left(\frac{\alpha_{1}}{\sin ^{2} \phi}+\frac{\alpha_{2}}{\cos ^{2} \phi}\right)
$$

with $G=\cos \theta$. For $m=2$ we have for the Hamiltonian $H_{2}^{0}$ the first integral

$$
U^{2} G=\cos \theta p_{u}^{2}-4 \frac{\sin \theta}{u} p_{\theta} p_{u}-4 \frac{\cos \theta}{u^{2}} p_{\theta}^{2}-4 \frac{\cos \theta}{u^{2} \sin ^{2} \theta}-8 \frac{\cos \theta}{u^{2}} V .
$$

\section{Extensions of TTW-type systems}

Let us consider the Hamiltonian

$$
L=\frac{1}{2} p_{1}^{2}+\frac{\zeta}{S_{\chi}^{2}\left(x_{1}\right)}\left(\frac{1}{2} p_{2}^{2}+F\left(x_{2}\right)\right), \quad \chi, \zeta \in \mathbb{R} \text { or } \mathbb{C} .
$$

For $\zeta=1, \chi$ real and

$$
F\left(x_{2}\right)=\frac{\alpha_{1}}{\cos ^{2} \lambda x_{2}}+\frac{\alpha_{2}}{\sin ^{2} \lambda x_{2}},
$$

$L$ is a generalization to constant curvature manifolds of the Tremblay-Turbiner-Winternitz system (see [7, 8]). We consider the possible extensions of $L$ in dimension three given by Theorem 1. Since the sectional curvature of the metric of $L$ is $\chi$, for $m c=\chi$ the general complete solution $G$ of $(2)$ is

$$
G=a_{0} C_{\chi}\left(x_{1}\right)+\left(a_{1} S_{\zeta}\left(x_{2}\right)+a_{2} C_{\zeta}\left(x_{2}\right)\right) S_{\chi}\left(x_{1}\right),
$$

and the extended Hamiltonian has the form

$$
H=\frac{1}{2} p_{u}^{2}+\frac{\chi}{S_{\kappa}^{2}\left(\frac{\chi}{m} u\right)} L,
$$

where we assume for simplicity $L_{0}=u_{0}=W_{0}=0$. Equation (3) becomes then

$$
F^{\prime}\left(a_{1} C_{\zeta}\left(x_{2}\right)-a_{2} \zeta S_{\zeta}\left(x_{2}\right)\right)=2 \zeta F\left(a_{1} S_{\zeta}\left(x_{2}\right)+a_{2} C_{\zeta}\left(x_{2}\right)\right) .
$$

If $a_{1}=a_{2}=0$, then the equation (14) is satisfied for all $F$, including the TTW potential with any value of $\lambda$. In particular, if $\lambda$ is rational then $L$ is superintegrable together with its extensions.

Otherwise, if $a_{1}$ or $a_{2}$ are different from zero then the solutions $F$ of the equation (14) can be obtained after observing that

$$
\zeta\left(a_{1} S_{\zeta}\left(x_{2}\right)+a_{2} C_{\zeta}\left(x_{2}\right)\right)=-\frac{d}{d x_{2}}\left(a_{1} C_{\zeta}\left(x_{2}\right)-a_{2} \zeta S_{\zeta}\left(x_{2}\right)\right) .
$$

Hence,

$$
F=\frac{1}{\left(a_{1} C_{\zeta}\left(x_{2}\right)-a_{2} \zeta S_{\zeta}\left(x_{2}\right)\right)^{2}} .
$$


By differentiating the relation $a_{1} S_{\zeta}\left(x_{2}\right)+a_{2} C_{\zeta}\left(x_{2}\right)=\mathcal{A} S_{\zeta}\left(x_{2}+\xi\right)$, valid for suitable constants $\mathcal{A}$ and $\xi$, we have

$$
F=\frac{1}{\mathcal{A}^{2} C_{\zeta}^{2}\left(x_{2}+\xi\right)},
$$

a result analogous to the one obtained in [1] for the extension of one-dimensional systems. Indeed, when $\zeta \in \mathbb{N} \backslash\{0\}$ then $L$ is in the form of an extension of a one-dimensional system. This is another example of iterative extension.

\section{Conclusions and future directions}

We have shown how the procedure of extension proposed in [1] can be used to produce new superintegrable systems starting from the already known ones, together with their first integrals. This procedure allows to extend a number of remarkable systems, including TTW and threeparticle Calogero systems. Moreover, in some cases the procedure can be performed iteratively, thus constructing a family of superintegrable systems in higher dimensions.

Unfortunately not all the superintegrable systems can be extended through our method, but this drawback is balanced by the simplicity and compactness of the algorithm that produces the constants of motion. Further studies are in progress to find a more general form of extension compatible with a larger number of potentials and to analyze iterative extension in other cases. New results about the application to nonconstant curvature manifolds of Theorem 1 have been obtained. The problem of applying the extension procedure to quantum systems is not yet solved: the first integrals described by Theorem 1 cannot be straightforwardly associated with symmetry operators. However, for the quadratic first integrals of type $U^{2}(G)$ a first quantization procedure has been considered, quite unsuccessfully, in [2] and then a second one has been studied, leading in suitable cases to symmetry operators. All these progresses will be presented in future publications.

\section{References}

[1] Chanu C., Degiovanni L., Rastelli G., First integrals of extended Hamiltonians in $n+1$ dimensions generated by powers of an operator, SIGMA 7 (2011), 038, 12 pages, arXiv:1101.5975.

[2] Chanu C., Degiovanni L., Rastelli G., Generalizations of a method for constructing first integrals of a class of natural Hamiltonians and some remarks about quantization, J. Phys. Conf. Ser. 343 (2012), 012101, 15 pages, arXiv:1111.0030.

[3] Chanu C., Degiovanni L., Rastelli G., Superintegrable three-body systems on the line, J. Math. Phys. 49 (2008), 112901, 10 pages, arXiv:0802.1353.

[4] Jauch J.M., Hill E.L., On the problem of degeneracy in quantum mechanics, Phys. Rev. 57 (1940), $641-645$.

[5] Kalnins E.G., Kress J.M., Pogosyan G.S., Miller Jr. W., Completeness of superintegrability in twodimensional constant-curvature spaces, J. Phys. A: Math. Gen. 34 (2001), 4705-4720, math-ph/0102006.

[6] Kalnins E.G., Kress J.M., Miller Jr. W., Talk given by J. Kress during the conference "Superintegrability, Exact Solvability, and Special Functions" (Cuernavaca, February 20-24, 2012).

[7] Kalnins E.G., Kress J.M., Miller Jr. W., Tools for verifying classical and quantum superintegrability, SIGMA 6 (2010), 066, 23 pages, arXiv:1006.0864.

[8] Maciejewski A.J., Przybylska M., Yoshida H., Necessary conditions for classical super-integrability of a certain family of potentials in constant curvature spaces, J. Phys. A: Math. Theor. 43 (2010), 382001, 15 pages, arXiv:1004.3854.

[9] Rodríguez M.A., Tempesta P., Winternitz P., Reduction of superintegrable systems: the anisotropic harmonic oscillator, Phys. Rev. E $\mathbf{7 8}$ (2008), 046608, 6 pages, arXiv:0807.1047.

[10] Tremblay F., Turbiner A.V., Winternitz P., An infinite family of solvable and integrable quantum systems on a plane, J. Phys. A: Math. Theor. 42 (2009), 242001, 10 pages, arXiv:0904.0738. 